\title{
Electrochemical Process and Phase Formation of Fe-Based Alloy Nanowires into Anodic Alumina Oxide
}

\author{
Tahir Mehmood ${ }^{1}$, Aiman Mukhtar ${ }^{1}$, Babar Shahzad Khan ${ }^{2}$, Adnan Saeed ${ }^{2}$, Waqar Ahmad ${ }^{3}$, \\ Wu kaiming, ${ }^{1, *}$ \\ ${ }^{1}$ The State Key Laboratory of Refractories and Metallurgy, Hubei Province Key Laboratory of \\ Systems Science in Metallurgical Process, International Research Institute for Steel Technology, \\ Wuhan University of Science and Technology, Wuhan 430081, P. R. China \\ ${ }^{2}$ Department of physics, Govt. College Women University, Sialkot, Punjab, Pakistan \\ ${ }^{3}$ Wuhan National Laboratory for Optoelectronics, Huazhong University of Science and Technology, \\ Wuhan 430074, P. R. China \\ *E-mail: tahir10621@yahoo.com, wukaiming@wust.edu.cn
}

doi: $10.20964 / 2017.02 .45$

Received: 14 October 2016 / Accepted: 19 November 2016 / Published: 30 December 2016

\begin{abstract}
The effect of deposition parameters on the phase formation and composition of Fe-Co alloy nanowires into nanopores of anodic alumina oxide membrane is considered by XRD, FE-SEM, TEM and EDX via electrochemical deposition technique. The deposited alloy nanowires are metastable fcc phase at $4.0 \mathrm{~V}$ and at $-2.0 \mathrm{~V}$ are stable hcp phase. These experimental results can be explained by the classic electrochemical nucleation theory. The formation of fcc crystals can be attributed to smaller critical clusters formed at a higher potential, lower deposition temperature and higher concentration of metal ions during electrochemical deposition process. The content of $\mathrm{Fe}$ inside nanowires increases with increasing potential, decreasing deposition temperature and increasing concentration of Fe ions. This can be verified by the polarization curves of depositing $\mathrm{Fe}$ and $\mathrm{Co}$ nanowires, where the current density ratio of $\mathrm{Co}$ to $\mathrm{Fe}$ at $-2.0 \mathrm{~V}(0.79)$ is higher than that at $-4.0 \mathrm{~V}(0.75)$.
\end{abstract}

Keywords: Nano cluster; electrochemical deposition; Crystal structure; Scanning electron microscopy; alloy nanowires;

\section{$\underline{\text { FULL TEXT }}$}

(C) 2017 The Authors. Published by ESG (www.electrochemsci.org). This article is an open access article distributed under the terms and conditions of the Creative Commons Attribution license (http://creativecommons.org/licenses/by/4.0/). 